ISSN: 2600-5859

\title{
El uso de Duolingo como herramienta de apoyo en el aprendizaje del Idioma Inglés
}

\section{The use of Duolingo as a Support Tool in English Language Learning}

\author{
Ángel Paul Obregón Mayorga. ${ }^{1}$, Mónica Alejandra Logroño Becerra. ${ }^{2}$ \& Carmita Eulalia \\ Rojas Castro. $^{3}$
}

Recibido: 20-11-2020 / Revisado: 26-12-2020 /Aceptado: 18-01-2021/ Publicado: 05-02-2021

\begin{abstract}
DOl: $\underline{\text { https://doi.org/10.33262/concienciadigital.v4i1.1.1557 }}$
\end{abstract}
This article explores the benefits of using the Duolingo mobile application in the classroom as a support tool in learning English as a second language in a course for students with a basic level (A1), at a public university in Ecuador. As today we know many institutions and teachers are using technology to teach their classes and it is more difficult to motivate and make students learn, for this reason, teachers are forced to look for new alternatives in order to be able to teach virtually in a single way. a way that is enjoyable for students. This article explains how Duolingo was used in English class, what activities can be done, and how it benefits students. Previous studies suggest that Duolingo is a very powerful application since this mobile application uses gamification as a learning technique, which allows students to learn through games which become challenges, creating healthy competition among students since all They compete by trying to complete the tasks, which are increased at different levels. This article also explains how the teacher assigns homework to his students and how he can keep track of each student.

Keywords: Gamification, Motivation, Autonomy, Duolingo, Mobile technology

1 Escuela Superior Politécnica de Chimborazo, Chimborazo, Ecuador, paul.obregon@espoch.edu.ec, https://orcid.org/0000-0001-6831-4848

${ }^{2}$ Escuela Superior Politécnica de Chimborazo, Chimborazo, Ecuador, ma_logrono@espoch.edu.ec, https://orcid.org/0000-0002-9348-8601

3 Escuela Superior Politécnica de Chimborazo, Chimborazo, Ecuador, crojasc@espoch.edu.ec, https://orcid.org/0000-0002-8247-0393 


\section{Resumen.}

El rol de la tecnología en el proceso enseñanza -aprendizaje, cada vez toma más fuerza en un entorno en el cual el dominio del conocimiento constituye la base fundamental para su desarrollo. En base a este principio, se han podido evidenciar un sinnúmero de aportes tecnológicos en muchas áreas del campo educativo, incluyendo el aprendizaje de una lengua extranjera. En lo que a la enseñanza-aprendizaje del idioma inglés concierne, es posible notar la amplia gama de herramientas tecnológicas con las cuales tanto docentes como estudiantes pueden contar para cumplir con sus objetivos y logros de aprendizaje de manera autónoma, didáctica y eficaz en una atmósfera atractiva y desafiante para los estudiantes, como por ejemplo la aplicación Duolingo. Este artículo explora los beneficios de usar esta aplicación en la clase como una herramienta de apoyo en el aprendizaje del idioma inglés como segunda lengua, en un curso de estudiantes con un nivel básico (A1), en una universidad pública de Ecuador. Además, detalla aspectos relevantes en el uso de Duolingo en la clase, y sus beneficios para el desarrollo de las diferentes destrezas comunicativas que el aprendizaje de una lengua extranjera requiere. Estudios anteriores señalan que Duolingo es un recurso didáctico de alto potencial ya que esta aplicación móvil se basa en la gamificación que es una técnica de aprendizaje cada vez más puesta en práctica, que permite a los estudiantes aprender a través de juegos los cuales se convierten en retos que desafían las destrezas de los estudiantes fortaleciendo su motivación y autonomía, lo cual fue posible evidenciar en los resultados del post test. Estos resultados además reflejan que existe un significativo grado de aceptación y actitud positiva de los estudiantes al competir y esforzarse por lograr sus objetivos completando los diferentes tipos de tareas enfocadas en las diferentes destrezas, cuyo grado de complejidad se incrementa gradualmente a medida que se superan los retos en los diferentes niveles. Este artículo también explica cómo el profesor asigna las tareas y cómo puede hacer un seguimiento a ellas.

Palabras claves: Gamificación, Motivación, Autonomía, Duolingo, Tecnología móvil

\section{Introducción.}

El avance de la tecnología es sin duda avasallante, cada día existen más innovaciones en este campo que requieren de una actualización constante para lograr nuestros objetivos con mayor rapidez y eficacia. En este sentido, la educación no es un ámbito que se aísla dentro de esta dinámica cambiante debido a la constante evolución de la pedagogía, especialmente en el contexto de inclusión de estos nuevos vientos que se advierten diversos, colaborativos y necesarios. (Castillo, n.d.). La educación sigue ahora una línea horizontal, en la que tanto el estudiante como el docente asumen roles equitativamente relevantes, cuya meta sigue siendo la misma, el aprendizaje efectivo, trascendente y significativo más aún ahora que las circunstancias habituales en las cuales se desarrollaban las diferentes actividades de nuestro entorno social han dado un giro drástico e inesperado debido a la reciente crisis sanitaria extendida a nivel mundial. 
En este contexto, el ámbito educativo también está experimentando ciertos cambios ya que la interacción directa entre docente y estudiante ha sido reemplazada por la interacción virtual haciendo que tanto docentes como estudiantes usen con mayor frecuencia las tecnologías de la información y comunicación (TIC'S) a través de los entornos virtuales de aprendizaje (EVAs) a fin de desarrollar los conocimientos y destrezas que deben ir a la par de una sociedad que demanda más competencias y aptitudes para su óptimo desarrollo, ya que facilitan en parte, el trabajo colaborativo a través de la comunicación sincrónica o asincrónica. (Arbeláez, 2014)

El uso de las TICS y aplicaciones en un entorno gamificado aportan significativamente en el área académica, y de allí la importancia del estudio de las diferentes aplicaciones gamificadas que se pueden implementar como herramientas de aprendizaje en las diferentes áreas de estudio. (Otazú Díaz, 2019). Esta afirmación se aplica en el aprendizaje de un idioma extranjero, en vista de que la gamificación como técnica educativa facilita y refuerza los procesos de asimilación y aprendizaje.

Para lograr este aprendizaje se cree pertinente el uso y aplicación de las TIC'S que lo analizaremos desde algunas perspectivas, como lo cita (López, 2013, pp 43,44) bajo una perspectiva del uso de las TIC'S en los procesos de enseñanza-aprendizaje:

Los estudiantes creen que algunas ventajas del uso de las TIC'S en el proceso son: menor tiempo de aprendizaje, acceso a múltiples recursos, personalización de la enseñanza y flexibilidad. Mientras que las desventajas son adicción, aislamiento, y mayor inversión de tiempo.

Los docentes en cambio consideran como ventajas la existencia de una mayor fuente de recursos educativos para la enseñanza, el contacto con los estudiantes, la facilidad en la evaluación y la actualización profesional, sin embargo, no hay que olvidar que también se puede obtener un mayor nivel de estrés, y que se pueden presentar dificultades en el mantenimiento de los equipos, además de la gran inversión de tiempo que requiere la planificación de sus actividades.

De forma general, si hacemos un análisis de esto tenemos que las ventajas dentro del proceso de aprendizaje son: el interés y la motivación generados, la interacción y actividad continua, fomenta de la imaginación, fortalece el aprendizaje colaborativo y la mejora la comunicación entre profesores y estudiantes, sin dejar de lado que también puede generar distracción, dispersión, ansiedad, y codependencia. (López, 2013, 43-44)

Los docentes encuentran en las TIC'S una gran cantidad de recursos educativos que a la vez que les permite actualizarse, también son un medio para establecer contacto con los estudiantes. Y a los estudiantes les permite escoger los recursos, una enseñanza flexible y 
personalizada, obteniendo como resultado un proceso lúdico, interesante y motivacional, en el que se fortalece el aprendizaje colaborativo y la comunicación.

Después de mirar ambas caras de la moneda, ventajas y desventajas del uso de las tecnologías de la información y comunicación con los resultados obtenidos del presente estudio se pretende promover el fortalecimiento de estrategias y técnicas innovadoras y atractivas como la gamificación a fin de motivar tanto a docentes como estudiantes a la consecución de sus logros de aprendizaje en la enseñanza del idioma inglés donde la gamificación como técnica metodológica, ha generado resultados favorables y alentadores en el proceso de enseñanzaaprendizaje contribuyendo al desarrollo de las diferentes destrezas comunicativas.

Pillaga, García, Cárdenas, y Erazo (2020), resaltan la relevancia de la gamificación como estrategia motivadora y dinámica para incrementar el nivel de inglés en los estudiantes creando una alianza didáctica entre aprendizaje y juego. El juego es una fuente de placer que, a través del aprendizaje significativo, permite a sus participantes aprender gracias a la motivación que genera. (Cea, 2018). Apreciaciones semejantes, han promovido a analizar la influencia de la aplicación Duolingo como una herramienta de apoyo en el aprendizaje del idioma inglés en estudiantes de nivel básico, al ser una herramienta versátil, sencilla, gratuita y accesible que se la puede usar a través de un computador o teléfono móvil.

Desde su aparición como aplicación gratuita, Duolingo ha sido ya merecedora del premio Apple a la mejor app en 2013 y ha sido objeto de varios estudios empíricos que avalan su calidad (Wagner \& KUNNAN, 2020) hecho que refuerza aún más las razones para el presente estudio, considerando además que su construcción se asienta en los principios de la gamificación; técnica en la que se refleja el aspecto lúdico, dentro del proceso de enseñanzaaprendizaje, este modelo consigue motivar intrínseca y extrínsecamente, (Sailer, n.d., 28-37). Dentro de la ludificación se diferencian algunas perspectivas que permiten una adecuada comprensión de la misma; como son las perspectivas del aprendizaje conductista, del interés y de la emoción, esta corriente pedagógica en auge desde hace unos años, aunque no demasiado adaptada todavía a la tecnología móvil (Jordano et al., 2013, 34) de estas aristas. El objetivo de la gamificación es también el de incentivar a los estudiantes hacia la superación personal y el logro de los objetivos.

Duolingo, se considera la aplicación de aprendizaje de idiomas más popular del mundo, que tiene más de 300 millones de usuarios (Smale, 2020,1) es así como a través de lecciones, fáciles y útiles se desarrollan las destrezas comunicativas para aprender inglés, de acuerdo con el nivel que tenga cada estudiante, lo que se puede determinar incluso con una prueba de nivel creada por la misma aplicación.

"Para muchas personas, aprender un idioma es una tarea difícil, y no tienen tiempo para inscribirse en clases formales semanalmente. Aplicaciones como Duolingo son una alternativa valiosa" (Smale, 2020, 1) lo cual sustenta el impacto positivo que el uso de esta 
herramienta como recurso alternativo puede generar en el aprendizaje de inglés resaltando su importancia como una aplicación complementaria con el fin de practicar diferentes actividades basadas en destrezas comunicativas como listening, speaking, reading and writing.

Otra de las ventajas que se pudo evidenciar al usar Duolingo, es el aprendizaje autónomo efectivo que esta aplicación promueve a través de la motivación. Para Benson (2015), los estudiantes que carecen de autonomía pueden potencializarla al ser expuestos a situaciones que les permitan tener el control sobre su propio proceso de aprendizaje. Esto se pudo evidenciar en el $70 \%$ de los estudiantes encuestados que reportaron un alto grado de aceptación de la aplicación como herramienta de apoyo para aprender inglés, así como el 90\% de estudiantes que reportó un alto grado de satisfacción con la aplicación.

Duolingo es un recurso complementario efectivo que hizo que los estudiantes sean los protagonistas de su propio aprendizaje al decidir cómo, cuándo, cuánto y dónde desarrollar las diferentes tareas presentadas en forma de retos y experiencias despertando el interés incluso de aquellos estudiantes con cierto rechazo o apatía en el aprendizaje de Inglés, quienes al tener mayor control sobre su tiempo de dedicación y esfuerzo, reportaron un incremento significativo en el desarrollo de sus destrezas.

Es importante resaltar que el uso de las aplicaciones gamificadas como en este caso Duolingo, pueden solventar o complementar las necesidades comunicativas e incluso reducir los niveles de rechazo y apatía en el aprendizaje de un idioma extranjero como se pudo evidenciar en la encuesta realizada. El impacto que Duolingo generó en los estudiantes fue altamente positivo, precisamente por sus sólidas bases en la técnica de la gamificación al atraer más estudiantes y asignarles un rol interactivo y participativo a través del uso de elementos del juego llevados a un ambiente opuesto (Ponce, 2015, como se citó en Nicholson,2012).

Las diferentes tareas que Duolingo presenta a través del juego; hace que éstas se tornen en retos que impulsan al desarrollo gradual de las destrezas de los estudiantes (Aguilera, Fúquene, y Ríos, 2014). Los resultados de este estudio demuestran el progreso de los estudiantes en el aprendizaje del idioma inglés lo cual, además, fue producto de su necesidad de lograr sus metas al sentirse desafiados por las diferentes tareas o experiencias, por lo que vale la pena resaltar una vez más el importante papel que juega la motivación en los estudiantes. Tal como Hammer (2007, como se citó en Gavarri 2016) hace referencia a la motivación como el resultado de una necesidad lo suficientemente poderosa que impulsa la decisión de actuar.

Duolingo es una herramienta fácil, entretenida y dinámica cuya configuración y estructura hace que las tareas o actividades sean presentadas de forma secuencial y organizada, esto además permite que el docente pueda efectuar un monitoreo y registro confiable del progreso del estudiante quien no puede avanzar al siguiente nivel sin antes cumplir los retos que 
comprometen el uso de sus habilidades. Todos estos beneficios que Duolingo ofrece, han hecho que el número de usuarios de esta aplicación sea cada vez más alto (Chik,Aoko and Smith 2018)

\section{Objetivos}

El Objetivo de este estudio es determinar si Duolingo es una aplicación efectiva como una herramienta de apoyo en el aprendizaje del idioma inglés como segunda lengua en un curso con estudiantes de nivel básico (A1).

Este estudio responde a las siguientes preguntas planteadas por el instructor.

- ¿Es Duolingo una herramienta fácil, divertida que ayuda a aprender inglés?

- ¿Es Duolingo una aplicación que promueve el aprendizaje de inglés durante el curso?

- ¿Es Duolingo una ayuda después de que se ha terminado el curso?

- ¿Duolingo promueve el aprendizaje individual?

\section{Metodologia.}

La población para este caso de estudio fue de 30 estudiantes con una edad promedio entre los de 18 y 19 años que se encontraban cursando primer semestre de una universidad pública en Ecuador con un nivel básico de inglés (A1). Para este estudio se utilizó Duolingo durante el periodo académico abril-septiembre del 2020. Como fase inicial de este estudio todos los estudiantes tomaron un pre-test gratuito que se encuentra disponible en la página de Duolingo. Para posteriormente registrarse en la página a través de un código de invitación a una clase previamente creada por el docente investigador usando la aplicación Duolingo for schools.

Los estudiantes podían registrarse utilizando su computador o su teléfono móvil y en vista de que todos tenían un nivel básico de inglés, se obvió el examen de ubicación que ofrecía Duolingo al momento de registrarse ya que, todos los estudiantes empezaron desde el nivel cero. Una vez registrados los estudiantes, el docente les asignaba desde la plataforma de Duolingo cinco actividades por semana, las cuales eran evaluadas como se detalla en la Tabla 1.

Tabla 1. Calificaciones de tareas asignadas en Duolingo

\begin{tabular}{cc}
\hline Tarea & Calificación \\
\hline 5 lecciones completadas & 10 puntos \\
4 lecciones completadas & 8 puntos
\end{tabular}




\begin{tabular}{ll}
3 lecciones completadas & 6 puntos \\
2 lecciones completadas & 4 puntos \\
1 lección completada & 2 puntos \\
\hline
\end{tabular}

Elaborado por: Grupo de Investigación

Nota. Estos valores fueron creados por el profesor investigador para evaluar las lecciones asignadas en Duolingo.

Cada semana los estudiantes completaron obligatoriamente cinco lecciones, pero estaban libres de completar más lecciones si querían hacerlo, esto, generó un poco de rivalidad entre los estudiantes ya que cada semana el profesor les indicaba el número de lecciones que habían terminado a la semana, los días que habían utilizado la aplicación, y el puntaje obtenido, este puntaje en Duolingo se llama experience. Muchas de las lecciones que el profesor había asignado a los estudiantes ya las habían terminado, esto fue muy bueno ya que los estudiantes debían repetirlas, con esto se logró que los estudiantes avancen, pero al mismo tiempo refuercen sus conocimientos en las lecciones repetidas. Duolingo también tiene una opción llamada strength meters la cual indica a los estudiantes qué destrezas en las lecciones tienen que revisarlas ya que los estudiantes tienden a olvidarlas con el transcurso del tiempo, y uno de los objetivos no fue hacer que los estudiantes avancen en las lecciones si no que practiquen más y de esta manera adquieran más puntos o experiencias como se los llama en Duolingo.

A medida que los estudiantes avanzaban, las lecciones se complicaban cada vez más ya que cada nivel tiene un grado más de dificultad, muchos de los estudiantes se sintieron frustrados ya que tenían que repetir las actividades hasta completar cada lección.

Al final del semestre los estudiantes tomaron el examen de práctica que ofrece Duolingo en su página web como Post-Test. Luego de este examen todos los estudiantes completaron una encuesta para medir el grado de satisfacción al usar Duolingo como una herramienta de ayuda en el aprendizaje del idioma inglés. Las preguntas se obtuvieron del estudio de eficacia de Duolingo realizado por (VASSELINOV \& Grego, 2012).

\section{Resultados.}

Además de las tareas asignadas cada semana, los estudiantes fueron motivados a tener un puntaje por los puntos (xp- Experiences) ganados cada semana. Al finalizar el semestre se obtuvo un reporte en Duolingo, donde se observa información cuantitativa sobre el número de días que los estudiantes utilizaron la aplicación (Days active), el número de lecciones concluidas (lessons) y el puntaje total obtenido por cada estudiante (xp - experiences). Ver Tabla 2.

Tabla 2. Puntaje detallado de las actividades realizadas en Duolingo. 
ISSN: 2600-5859

Vol. 4, N¹.1, p. 250-266, Febrero, 2020

\begin{tabular}{|c|c|c|c|c|c|}
\hline No & Username & Language & $\begin{array}{c}\text { Days } \\
\text { Active }\end{array}$ & Lessons & $\mathbf{X P}$ \\
\hline 1 & MY & ENGLISH & 50 & 327 & 7051 \\
\hline 2 & SP & ENGLISH & 17 & 110 & 1598 \\
\hline 3 & $\mathrm{AO}$ & ENGLISH & 27 & 232 & 8696 \\
\hline 4 & FA & ENGLISH & 20 & 127 & 5803 \\
\hline 5 & $\mathrm{EV}$ & ENGLISH & 70 & 344 & 10556 \\
\hline 6 & LED & ENGLISH & 61 & 443 & 12349 \\
\hline 7 & FLL & ENGLISH & 23 & 198 & 6331 \\
\hline 8 & DF & ENGLISH & 19 & 104 & 6145 \\
\hline 9 & ANM & ENGLISH & 15 & 133 & 4461 \\
\hline 10 & APC & ENGLISH & 10 & 61 & 1156 \\
\hline 11 & CEV & ENGLISH & 24 & 253 & 3644 \\
\hline 12 & HIM & ENGLISH & 31 & 190 & 2647 \\
\hline 13 & FJL & ENGLISH & 11 & 138 & 2037 \\
\hline 14 & JCS & ENGLISH & 11 & 132 & 1989 \\
\hline 15 & $\mathrm{JC}$ & ENGLISH & 15 & 145 & 2194 \\
\hline 16 & EG & ENGLISH & 13 & 60 & 1103 \\
\hline 17 & $\mathrm{EC}$ & ENGLISH & 13 & 84 & 1422 \\
\hline 18 & $\mathrm{AMZ}$ & ENGLISH & 20 & 239 & 3492 \\
\hline 19 & $\mathrm{JB}$ & ENGLISH & 53 & 152 & 2854 \\
\hline 20 & PL & ENGLISH & 11 & 108 & 1740 \\
\hline 21 & $\mathrm{KG}$ & ENGLISH & 8 & 101 & 1526 \\
\hline 22 & $\mathrm{RC}$ & ENGLISH & 12 & 101 & 1464 \\
\hline 23 & $\mathrm{KS}$ & ENGLISH & 6 & 23 & 413 \\
\hline 24 & $\mathrm{NCH}$ & ENGLISH & 13 & 147 & 2136 \\
\hline 25 & $\mathrm{JN}$ & ENGLISH & 19 & 143 & 2072 \\
\hline 26 & SPN & ENGLISH & 13 & 163 & 2390 \\
\hline 27 & AS & ENGLISH & 8 & 109 & 1650 \\
\hline 28 & $\mathrm{LD}$ & ENGLISH & 20 & 184 & 2903 \\
\hline 29 & $\mathrm{KFC}$ & ENGLISH & 12 & 152 & 2167 \\
\hline 30 & VUU & ENGLISH & 13 & 135 & 2045 \\
\hline
\end{tabular}

Elaborado por: Grupo de Investigación

Nota. Estos valores se obtuvieron del detalle del reporte de actividades de Duolingo.

Como se mencionó anteriormente, los estudiantes acumulaban el puntaje obtenido cada semana, y al final del semestre este puntaje (XP- experiences) tuvo un valor de 5 puntos. Esta nota se calculó con una regla de tres, tomando como base el máximo puntaje obtenido. Por ejemplo, como se evidencia en la Tabla 2, el estudiante LED obtuvo 12349 experiences de puntaje, lo cual representa un valor de 5 puntos. 
Los resultados obtenidos en la encuesta para determinar el grado de satisfacción del uso de Duolingo como una herramienta de apoyo en el aprendizaje del idioma inglés se pueden ver en la Tabla 3.

Tabla 3. Grado de satisfacción del uso de Duolingo.

\begin{tabular}{|c|c|c|c|c|c|}
\hline \multicolumn{6}{|c|}{ ¿Fue Duolingo fácil de usar? } \\
\hline $\mathbf{n} .=30$ & $\begin{array}{l}\text { Totalmente en } \\
\text { Desacuerdo }\end{array}$ & $\begin{array}{l}\text { En } \\
\text { Desacuerdo }\end{array}$ & $\begin{array}{l}\text { Ni de acuerdo, ni } \\
\text { en Desacuerdo }\end{array}$ & $\begin{array}{l}\text { De } \\
\text { Acuerdo }\end{array}$ & $\begin{array}{l}\text { Totalmente } \\
\text { de Acuerdo }\end{array}$ \\
\hline $\begin{array}{l}\text { ¿Fue Duolingo fácil de } \\
\text { usar? }\end{array}$ & $0 \%$ & $3.3 \%$ & $23.3 \%$ & $33.3 \%$ & $40 \%$ \\
\hline $\begin{array}{l}\text { ¿Fue Duolingo de } \\
\text { ayuda aprendiendo } \\
\text { inglés? }\end{array}$ & $0 \%$ & $3.3 \%$ & $6.7 \%$ & $40 \%$ & $50 \%$ \\
\hline $\begin{array}{l}\text { ¿Disfrute aprendiendo } \\
\text { inglés con Duolingo? }\end{array}$ & $3.3 \%$ & $3.3 \%$ & $16.7 \%$ & $36.7 \%$ & $40 \%$ \\
\hline $\begin{array}{l}\text { ¿Estoy satisfecho con } \\
\text { Duolingo? }\end{array}$ & $3.3 \%$ & $6.7 \%$ & $20 \%$ & $36.7 \%$ & $33.3 \%$ \\
\hline
\end{tabular}

Elaborado por: Grupo de Investigación

Nota. Estos valores se obtuvieron de la encuesta de satisfacción realizada a los estudiantes al finalizar el curso.

La encuesta realizada arrojó los siguientes resultados: En la primera pregunta, ¿Fue Duolingo fácil de usar?, las opciones de Acuerdo y Totalmente de Acuerdo reportan que Duolingo es una herramienta sencilla, accesible y amigable para el uso de los estudiantes, ya que, al sumar los valores obtenidos, se puede verificar que el $73.32 \%$, encontró Duolingo fácil de usar, esto significa que de un total de 30 estudiantes 22 de ellos pudieron acceder a la aplicación con facilidad. Respecto a la misma pregunta, el 23.3\% de estudiantes reportó Ni de Acuerdo ni en Desacuerdo, esto significa que 7 estudiantes mantuvieron una posición neutral respecto a la facilidad de uso de Duolingo, mientras que un estudiante reportó estar en desacuerdo respecto a este punto, lo cual representa un $3.3 \%$. No hubo reportes en la opción Totalmente en Desacuerdo lo que significa que todos los estudiantes lograron acceder a la aplicación. Comprobando así que Duolingo posee características que la hacen fácil de usar

En la segunda pregunta: ¿Fue Duolingo de ayuda aprendiendo inglés?, se observa que 15 estudiantes están Totalmente de Acuerdo y 12 estudiantes están De Acuerdo, esto suma 27 estudiantes con un porcentaje positivo de $90 \%$ frente a un $6.7 \%$ que se mantuvo en la posición ni acuerdo ni en desacuerdo, es decir, que de un total de 30 estudiantes dos de ellos, reportaron no haber experimentado ningún cambio positivo o negativo en su proceso de aprendizaje. Las opciones en desacuerdo y Totalmente en desacuerdo. tienen una variación entre uno y cero estudiantes respectivamente, lo cual revela que un porcentaje de entre el $3.3 \%$ y $0 \%$ no considera a Duolingo como una herramienta de ayuda para aprender inglés. 
Los resultados de esta pregunta demuestran la efectividad de esta herramienta como apoyo para incrementar el nivel de inglés.

En la tercera pregunta: ¿Disfruté aprendiendo inglés con Duolingo?, se observa que 12 estudiantes están Totalmente de Acuerdo y 11 estudiantes están de Acuerdo, dando un total de 23 estudiantes, lo que representa un porcentaje positivo de $76.7 \%$ del total de estudiantes encuestados que disfrutaron con las diferentes tareas de esta aplicación durante su proceso de aprendizaje. Este hecho, permite comprobar que el carácter lúdico de la aplicación es de gran importancia para los estudiantes en el proceso de aprendizaje. Cinco estudiantes mantuvieron la posición ni en Acuerdo ni en Desacuerdo, lo que da un porcentaje de 16.7\%. Un estudiante estuvo en desacuerdo y otro estudiante Totalmente en Desacuerdo, los dos porcentajes de $3.33 \%$ suman un porcentaje total de $6.66 \%$ que estuvieron en Desacuerdo y Total Desacuerdo que disfrutaron aprendiendo inglés con Duolingo.

En la cuarta y última pregunta: ¿Estoy satisfecho con Duolingo?, se observa que 10 estudiantes están Totalmente de Acuerdo y 11 estudiantes están de Acuerdo, lo que suma 21 estudiantes correspondiente al $70 \%$ de los estudiantes encuestados, quienes reportaron su satisfacción con el uso de Duolingo. Seis estudiantes mantuvieron la postura ni De acuerdo ni en acuerdo representando el $20 \%$ mientras que dos estudiantes están en Desacuerdo dando un porcentaje de $6.7 \%$ mientras que un estudiante que representa el $3.33 \%$ está en Total Desacuerdo, ambos porcentajes suman un total de $10 \%$ de estudiantes que estuvieron en Desacuerdo y Total Desacuerdo respecto al hecho de estar satisfechos con Duolingo.

En la pregunta número 2 ¿Recomendarías Duolingo a un compañero o amigo?, se obtuvo la siguiente información. Ver Tabla 4.

Tabla 4. Recomendación de Duolingo a un compañero o amigo

\begin{tabular}{|c|c|c|c|c|c|c|c|c|c|c|c|c|}
\hline \multirow{2}{*}{$\begin{array}{l}\text { 10) } \\
n=30\end{array}$} & \multicolumn{12}{|c|}{ ¿Recomendarías Duolingo a un compañero o amigo? (en una escala de 0 a } \\
\hline & & & & & & & & & & & & \\
\hline $\begin{array}{l}\text { Muy } \\
\text { improbable }\end{array}$ & 0 & 1 & 2 & 3 & 4 & 5 & 6 & 7 & 8 & 9 & 10 & $\begin{array}{l}\text { Muy } \\
\text { probable }\end{array}$ \\
\hline & & & & & & & $3 \%$ & $33 \%$ & $40 \%$ & $43.33 \%$ & $10 \%$ & \\
\hline
\end{tabular}

Elaborado por: Grupo de Investigación

Nota. Estos valores se obtuvieron de la encuesta de satisfacción realizada a los estudiantes al finalizar el curso.

Como podemos ver los valores en la Tabla 4. Los estudiantes recomiendan Duolingo a un compañero o amigo de la siguiente manera. Un total de 3 estudiantes con un porcentaje de $10 \%$ recomiendan el uso de Duolingo con un valor Muy probable de 10 puntos. Trece 
estudiantes con un porcentaje de $43.33 \%$ recomiendan el uso de Duolingo con un valor Muy probable de 9 puntos. Doce estudiantes con un porcentaje de $40 \%$ recomiendan el uso de Duolingo con un valor Muy probable de 8 puntos. Un estudiante con un porcentaje de 3.33\% recomienda el uso de Duolingo con un valor Muy probable de 7 puntos. Finalmente, un estudiante con un porcentaje de $3.33 \%$ recomienda el uso de Duolingo con un valor probable de 6 puntos.

Los resultados obtenidos en la pregunta 3 ¿Te gustó más las tareas en Duolingo que las tareas regulares? Se pueden observar en la Tabla 5.

Tabla 5. Comparación de Duolingo con las tareas regulares.

\begin{tabular}{lccc}
\hline \multicolumn{4}{c}{ ¿Te gustó más las tareas en } \\
\hline Estudiantes (n.=30) & Yes & No & Igual \\
& $70 \%$ & $16.7 \%$ & $13.3 \%$ \\
\hline
\end{tabular}

Elaborado por: Grupo de Investigación

Nota. Estos valores se obtuvieron de la encuesta de satisfacción realizada a los estudiantes al finalizar el curso.

Como podemos observar los valores en la Tabla 5, al 70\% de estudiantes les gustó más las tareas en Duolingo que las tareas regulares, esto es un total de 21 estudiantes. A 5 estudiantes, lo cual es el $16.7 \%$ les gustó más las tareas regulares, y a 4 estudiantes lo que representa el $13.3 \%$ les dio igual hacer las tareas en Duolingo y las tareas ordinarias.

Los resultados obtenidos en la pregunta 4 ¿Cómo ingresaste a Duolingo? se pueden observar en el Gráfico 1.

De un total de 30 estudiantes, 22 estudiantes con un porcentaje de $73.3 \%$ ingresaron a Duolingo a través de sus teléfonos móviles, lo que parece ser lo más óptimo, ya que los estudiantes siempre están utilizando su teléfono móvil, 0.5 estudiantes con un porcentaje de 16.7 ingresaron a Duolingo desde sus computadoras, y estudiantes con un porcentaje de $10 \%$ ingresaron a Duolingo desde una Tablet. 
Figura 1. Acceso a Duolingo

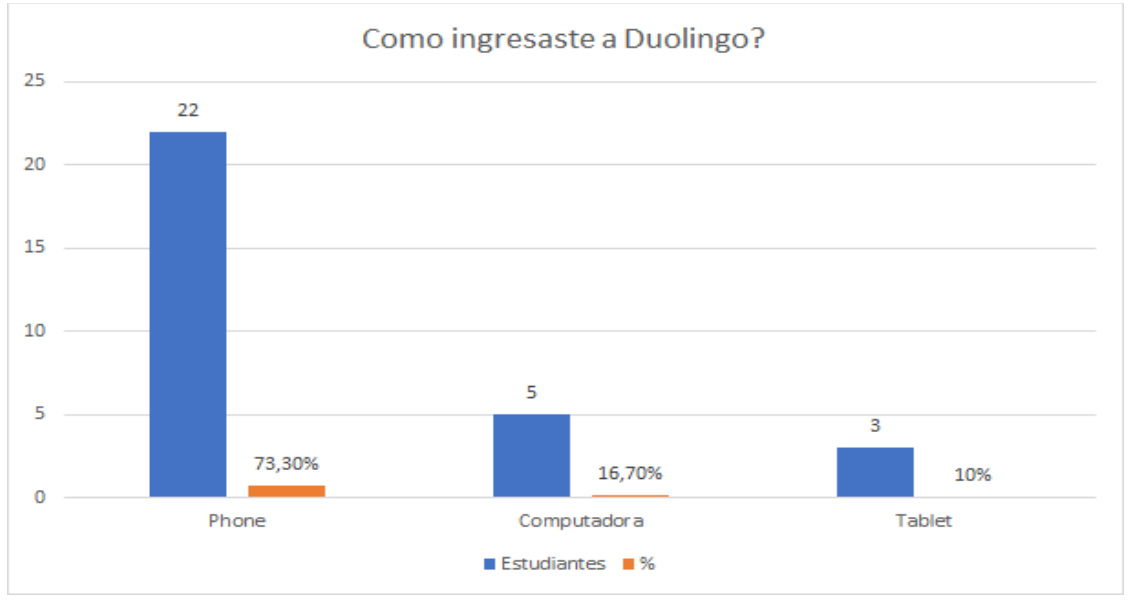

Nota. El gráfico representa los porcentajes de cómo los estudiantes ingresaron a Duolingo, las opciones fueron, por teléfono, computadora y Tablet. Esta información se obtuvo de la encuesta de satisfacción hecha por los estudiantes al final del curso.

Finalmente podemos observar en la Tabla 6 , los datos obtenidos en la pregunta 5 . ¿Continuarías usando Duolingo?

Tabla 6. Duolingo después de terminado el curso

\begin{tabular}{lccc}
\hline \multicolumn{4}{c}{ ¿Continuarías usando Duolingo? } \\
\hline $\mathbf{N}=\mathbf{3 0}$ & $\mathbf{S i}$ & No & Tal vez \\
& $83.3 \%$ & $3.3 \%$ & $13.3 \%$ \\
\hline
\end{tabular}

Elaborado por: Grupo de Investigación

Nota. Estos valores se obtuvieron de la encuesta de satisfacción realizada a los estudiantes al finalizar el curso.

Como podemos observar en la Tabla 6, 25 estudiantes lo cual es el 83.3\% dijo que continuará usando Duolingo después de terminado el curso, sin embargo 1 estudiante, lo cual es el $3.3 \%$ dijo que no va a utilizar Duolingo después de terminado el curso. 4 estudiantes es decir el 13.3\% dijeron que tal vez utilizarían Duolingo después de terminado el curso.

Los datos obtenidos en el Pre Test y Post Test del examen de Duolingo los podemos observar en la Tabla 7. Según la página web de Duolingo, los puntajes del examen entre 10 y 55 puntos, significa que los estudiantes pueden comprender y entender frases muy básicas y también expresarse en contextos familiares. Y el puntaje entre 60 y 85 puntos, significa que los estudiantes pueden comprender los puntos principales de un discurso o escritura concreta 
ISSN: 2600-5859

sobre asuntos rutinarios como el trabajo y la escuela, también puede describir experiencias, ambiciones, opiniones y planes, aunque con cierta incomodidad o dudas.

Los resultados obtenidos en el Post Test muestran que hay un incremento en el resultado del Post Test, lo cual quiere decir que el uso de Duolingo es un buen aporte como una herramienta de apoyo en el aprendizaje de inglés.

Tabla 7. Comparación del Pre-Test y Post Test de Duolingo

\begin{tabular}{|c|c|c|}
\hline$n=30$ & Pre-Test & Post Test \\
\hline MY & 25 & 40 \\
\hline SP & 15 & 45 \\
\hline $\mathrm{AO}$ & 27 & 48 \\
\hline FA & 30 & 68 \\
\hline $\mathrm{EV}$ & 10 & 39 \\
\hline LED & 38 & 61 \\
\hline FLL & 45 & 65 \\
\hline $\mathrm{DF}$ & 12 & 41 \\
\hline ANM & 14 & 39 \\
\hline APC & 17 & 49 \\
\hline CEV & 35 & 63 \\
\hline HIM & 37 & 56 \\
\hline FJL & 39 & 68 \\
\hline JCS & 20 & 46 \\
\hline $\mathrm{JC}$ & 19 & 52 \\
\hline EG & 14 & 53 \\
\hline $\mathrm{EC}$ & 13 & 43 \\
\hline AMZ & 12 & 40 \\
\hline $\mathrm{JB}$ & 11 & 41 \\
\hline PL & 13 & 45 \\
\hline $\mathrm{KG}$ & 40 & 67 \\
\hline $\mathrm{RC}$ & 32 & 58 \\
\hline KS & 34 & 59 \\
\hline $\mathrm{NCH}$ & 39 & 63 \\
\hline $\mathrm{JN}$ & 14 & 46 \\
\hline SPN & 31 & 60 \\
\hline AS & 32 & 67 \\
\hline $\mathrm{LD}$ & 23 & 52 \\
\hline $\mathrm{KFC}$ & 21 & 50 \\
\hline VUU & 24 & 45 \\
\hline
\end{tabular}

Elaborado por: Grupo de Investigación

Nota. Estos datos son las notas de los estudiantes del Pre Test y Post Test 


\section{Discusión}

El uso de Duolingo como una herramienta de apoyo en el aprendizaje de inglés, se considera que es positivo ya que esta herramienta permitió a los estudiantes mejorar sus destrezas de inglés, en un ambiente divertido y competitivo. Los resultados obtenidos de la satisfacción del uso de Duolingo muestran que esta herramienta tiene muchas características positivas, por ejemplo, crea en los estudiantes una competencia sana y los motivó a utilizar más esta aplicación con el fin de ganar puntaje (XP - experiencias). Esto fue muy importante ya que mientras más puntaje o experiencias ganaban, más tiempo practicando inglés tenían, los estudiantes repetían las actividades una y otra vez hasta pasar de nivel, esta repetición fue positiva para ampliar el vocabulario de los estudiantes.

Basándonos en los resultados de la encuesta podemos decir que los estudiantes encontraron a Duolingo como una herramienta fácil de usar, que fue de mucha ayuda y que disfrutaron aprender inglés usando esta herramienta, se puede decir también que las tareas en Duolingo fueron más divertidas que las tareas regulares ya que los estudiantes podían practicar y hacer su tarea directamente desde sus teléfonos móviles, que fue el dispositivo más utilizado al momento de practicar inglés en Duolingo. Los estudiantes si recomiendan usar Duolingo a amigos y compañeros de estudio, además piensan utilizar Duolingo después de terminar el curso ya que es una herramienta en la cual pueden practicar todas las destrezas del idioma inglés.

Para el investigador el uso de Duolingo fue una herramienta práctica, que le permitió fácilmente hacer un seguimiento de las actividades asignadas a los estudiantes, Duolingo permite generar un reporte del puntaje (XP - experiencias) obtenido por los estudiantes. Este reporte muestra el número de días en que el estudiante utilizó Duolingo, el número de lecciones completadas, así como también el puntaje ganado.

\section{Conclusiones.}

- Este estudio sugiere utilizar Duolingo como una herramienta de apoyo en un curso de inglés, porque promueve el aprendizaje individual de los estudiantes ya que lo pueden hacer en cualquier momento y lugar desde sus teléfonos móviles. Duolingo promueve el aprendizaje individual ya que crea una competencia sana entre estudiantes, que se ve reflejada en los puntajes alcanzados y en el número de días que la aplicación ha sido utilizada.

- Duolingo es una aplicación que utiliza la gamificación como una técnica de aprendizaje, y por esta razón es que los estudiantes invierten mucho tiempo utilizándola para practicar su inglés, ya que casi todos los estudiantes, mencionaron que van a seguir utilizando Duolingo después de terminado el curso, y que incluso la recomendarían a otras personas, ya que ellos la consideran como una herramienta muy útil, fácil de usar y muy interactiva para aprender inglés. 
- Los resultados del Post Test indican que las calificaciones obtenidas en el examen de Duolingo al finalizar el curso son mayores que los obtenidos en el Pre Test, por consiguiente, concluimos que Duolingo es una herramienta que motiva a los estudiantes a aprender y practicar en una forma más divertida, esto se refleja en los resultados del Post Test y en los resultados de satisfacción de los estudiantes al usar Duolingo.

- También concluimos que el uso de la tecnología en el aprendizaje de inglés facilita tanto a estudiantes como a profesores, ya que existen herramientas que son de mucha ayuda, por sus técnicas de aprendizaje, como es el uso de la gamificación en Duolingo y que pueden ser utilizadas en cualquier lugar mediante un dispositivo electrónico, lo cual convierte esas tareas tradicionales en divertidas, motivando al estudiante a practicar más y por ende a mejorar su nivel de inglés.

\section{Referencias bibliográficas.}

Aguilera Castillo, A., Fúquene Lozano, C. A., \& Ríos Pineda, W. F. (2014, Junio 14). Aprende jugando: El uso de Técnicas de Gamificación en Entornos de Aprendizaje. IM-Pertinente, $2(1)$, 125-143. https://www.researchgate.net/profile/William_Rios2/publication/317083719_Apren de_jugando_el_uso_de_tecnicas_de_gamificacion_en_entornos_de_aprendizaje/lin ks/592497ffaca27295a8c0f1f4/Aprende-jugando-el-uso-de-tecnicas-degamificacion-en-entornos-de-aprendiz

Arbeláez, M. (2014, Julio Lunes). Las tecnologías de la información y la comunicación(TIC) un instrumento para la investigación. http://www.scielo.org.co/scielo.php?script=sci_arttext\&pid=S012481462014000200001

Benson, P. (Routledge, 2015). Teaching and Researching: Autonomy in Language Learning Applied Linguistics in Action Series. Routledge.

Castillo, M. (n.d.). Desafíos de la educación en la sociedad actual. Revista electrónica.

Cea, F. (2018, diciembre). Motiva a Tus Alumnos Con El Aprendizaje a Través Del Juego. AulaPlaneta. https://www.aulaplaneta.com/2018/12/14/educacion-y-tic/motiva-atus-alumnos-con-el-aprendizaje-a-traves-del-juego/

Chik, A., Aoki, N., \& Smith, R. (2018). Autonomy in LAnguage Learning and Teaching. New Research Agendas.

Gavarri, S. L. (2016, Abril). El aprendizaje de lenguas extranjeras mediado por las TIC: Aprender Inglés con Duolingo. El Toldo de Astier, 7(12), 56-65. http://www.memoria.fahce.unlp.edu.ar/art_revistas/pr.7199/pr.7199.pdf

Jordano, M., Castrillo, M., \& Pareja, A. (2013). El aprendizaje de lenguas extranjeras mediante tecnología móvil en el contexto de la educación a distancia y combinada. Revista Iberoamericana de Educación a Distancia, 19(1), 25-40. https://www.redalyc.org/pdf/3314/331443195002.pdf 
ISSN: 2600-5859

López, M. (2013). ¿Qué piensan los futuros maestros? In Las nuevas tecnologías en el proceso de enseñanza aprendizaje (pp. 40-61). Tejuelo.

Otazú Díaz, V. M. (2019, Agosto 7-9). La gamificación y uso de TIC’S en el aula como recursos de una metodología innovadora y motivadora para el aprendizaje del inglés técnico implementado en el tercer semestre de la carrera de nutrición humana de la Universidad del Pacifico, marzo-julio de 20. RESÚMENES DEL II CONGRESO INTERNACIONAL DE INNOVACIÓN EN EDUCACIÓN EN CIENCIAS DE LA $S A L U D, 34-35$. https://www.upacifico.edu.py/img/resumen_congreso.pdf\#page=36

Pillaga Guamán, L., Garcia Herrera, D. G., Cárdenas Cordero, N. M., \& Erazo Álvarez, J. C. (2020, Junio 29). Alianza entre Aprendizaje y Juego: Gamificación Como Estrategia Metodológica que Motiva el Aprendizaje del Inglés. Revista Arbitrada Interdisciplinaria KOINONIA, V(1), 388. http://dx.doi.org/10.35381/r.k.v5i1.788

Ponce Ortigón, E. (2015, Marzo). Gamification como estrategia para aumentar el student engagement en estudiantes de secundaria y preparatoria. Retrieved Enero 27, 2021, from https://d1wqtxts1xzle7.cloudfront.net/39509301/engagementbecario.pdf?1446074486=\&response-contentdisposition=inline\%3B+filename\%3DGamification_como_estrategia_para_aument. pdf \&Expires $=1611787042 \&$ Signature=TUT9owQ2QqOkBILowHDEr5EmwcSYq moWUdliRS9jYnfLbGzunI

Sailer, M., Mandl, J., \& Klevers, M. (n.d.). Psychological perspectives on motivation through gamification. Interaction Design and Architecture(s) Journal, (19).

Smale, W. (2020, 01 27). Duolingo: Luis von Ahn, el latinoamericano que le enseña un nuevo idioma a 300 millones de personas. bbc news. https://www.bbc.com/mundo/noticias51241014

VASSELINOV, R., \& Grego, J. (2012). Duolingo(Effectiveness(Study. Static Duolingo. https://static.duolingo.com/s3/DuolingoReport_Final.pdf

Wagner, E., \& KUNNAN, A. (2020). The Duolingo English Test. Language Assesment Quaterly. 10.1080/15434303.2015.1061530

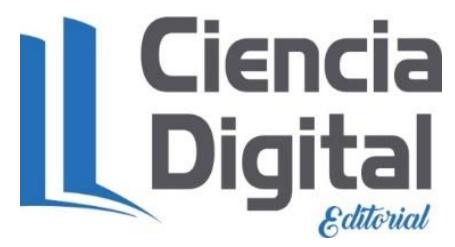


PARA CITAR EL ARTÍCULO INDEXADO.

Obregón Mayorga, Ángel P., Logroño Becerra, M. A., \& Rojas Castro, C. E. (2021). El uso de Duolingo como herramienta de apoyo en el aprendizaje del Idioma Inglés . ConcienciaDigital, 4(1.1), 250-266. https://doi.org/10.33262/concienciadigital.v4i1.1.1557

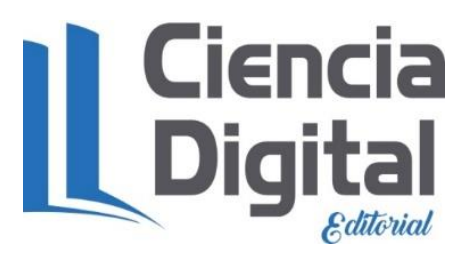

El artículo que se publica es de exclusiva responsabilidad de los autores y no necesariamente reflejan el pensamiento de la Revista Conciencia Digital.

El artículo queda en propiedad de la revista y, por tanto, su publicación parcial y/o total en otro medio tiene que ser autorizado por el director de la Revista Conciencia Digital.

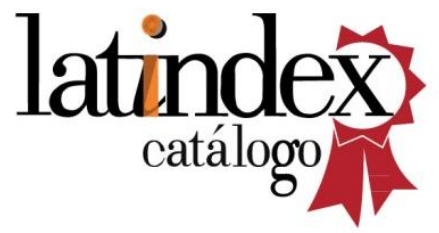

Conciencia

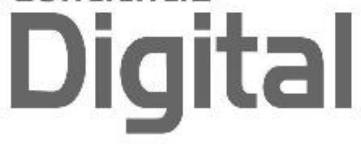

